\title{
Study of Ovarian Neoplastic Lesions
}

\author{
Annapurna Parvatala ${ }^{1}$, Ragidi Ramesh Babu ${ }^{2}$, Natta Bharat Rao ${ }^{3}$, \\ K. Narasimhulu ${ }^{4}$ \\ ${ }^{I}$ (Assistant Professor Of Pathology, Siddhartha Medical College, Vijayawada, India) \\ ${ }_{2}^{2}$ (Assistant Professor Of Radiotherapy, Siddhartha Medical College, Vijayawada, India) \\ ${ }_{3}^{3}$ (Professor Of Pathology, Siddhartha Medical College, Vijayawada, India) \\ ${ }^{4}$ (Senior Resident, Dept. Of Pathology, Siddhartha Medical College, Vijayawada, India)
}

\begin{abstract}
Ovaries are paired structures with an embryological, anatomic and functional complexity. Changes in their volume can be observed at ovulation, pregnancy and after menopause. Ovaries are normally resistant to diseases, but still ovary can be seat of more number of neoplastic lesions of benign and malignant. They may be primary or secondary, large or small, solid or cystic and also of mixed patterns and histological types of a broad spectrum. Ovaries are hormone influenced organs and so make a base for hormone related diseases. At the same time many other hormonally unrelated lesions are also seen in ovaries. They are asymptomatic and physical examination is difficult in early stages and hence ovarian neoplastic lesions are difficult to be diagnosed early. The present study is focused on parameters like incidence, bilaterality, age distribution, and spectrum of ovarian neoplasms. The risk of development of neoplasm in hormone influenced organs like breast and uterus is increased with hormone influenced ovarian neoplasms. The wide spectrum of ovarian neoplasms includes harmless simple cystic lesions to the fatal aggressive malignant lesions. The present study is focused on the incident rates of age, site and distribution of the various ovarian neoplastic lesions.
\end{abstract}

Keywords: Neoplastic Lesions, Granulosa cell tumor, Mature cystic teratoma, Yolk sac tumor, Krukenberg Tumor, surgical excision, chemotherapy, radiotherapy.

\section{Introduction}

Ovarian neoplasms pose a big challenge to the gynaecologist in finding out the causation of Cancer deaths. Available knowledge of the etiological factors is less and the oncologist may not achieve any considerable reduction in the mortality rate. The pathologist is often frustated by the numerous neoplastic entities hosted by ovary and by so many histogenetic theories and to explain the occurrences. Ovarian neoplasms represent around 30\% of all malignancies of the female genital system[1]. In the economically advanced countries, age adjusted incidence rates are high. Surface epithelial-stromal carcinomas contribute for 90\% of all ovarian cancers in North America and Western Europe. In Japan like Asian countries, Germ cell tumours account for a considerable proportion (20\%) of ovarian cancers. High parity and the usage of oral contraceptives are associated with a reduced risk of developing surface epithelial-stromal tumours, whereas prolonged estrogen replacement therapy increases the risk in postmenopausal women[1].

Ovarian cancer is the third most frequent malignancy with a higher mortality. By the time of diagnosis itself majority of the patients have tumour spread in the pelvis or abdomen. Regular ultrasound monitoring \& bimanual examination of all women and also regular follow up in peri/postmenopausal women is helpful to detect early and to reduce the mortality of ovarian tumours. In the ovarian neoplasms with endocrine activity, which cause various clinical symptoms and signs, and in some feminizing ovarian tumours associated with endometrial carcinoma the problem is much more complicated. As the ovary is targeted to various hormones from menarche to menopause and repeated involutions there by prone to tumour formation. According to Silverberg grading is more helpful in predicting tumour response to chemotherapy and acts as a guide for therapeutic protocols. Histological typing is more useful than grading in predicting survival[2].

The present study comprised of 880 ovarian lesions, received during the study period of three years in the Pathology department, Siddhartha Medical College, Vijayawada. Out of 880 ovarian lesions 92 lesions were observed as neoplastic lesions $(10.45 \%$ ) which were included as study material. Bilateral incidence was $30.43 \%$ (28 of 92) and unilateral incidence was $69.57 \%$ (64 of 92) in neoplastic lesions. Age incidence varied from $21 \mathrm{yrs}$ to $65 \mathrm{yrs}$ with a peak between $31 \mathrm{yrs}$ to $50 \mathrm{yrs}$ age group (71\%). Most of the lesions (more than $80 \%$ ) were in the group of $21 \mathrm{yrs}$ to $50 \mathrm{yrs}$. Benign lesions were observed in earlier (21yrs to 50yrs) when compared to the malignant lesions (31yrs to 60yrs). Surface epithelial tumours contributed major share of the neoplastic lesions, in both benign and malignant classifications. Serous cystadenomas are the commonest benign lesions reported (47.7\%), whereas surface epithelial malignant lesions contributed $66.67 \%$ of that group. Serous cystadeno carcinomas were the commonly reported malignant tumors. Still a considerable number (646) of ovaries had normal histologcal picture and often no specific pathological changes noted. 


\section{Materials and Methods}

Study period: three years

Study design: Prospective, Cross sectional and observational

Study material: all ovarian neoplastic specimens received during the study period in department of Pathology, Siddhartha Medical College, Vijayawada.

Inclusion: all the ovarian specimens which are diagnosed as neoplastic lesions received during study period in department of Pathology, SMC, Vijayawada.

Exclusion: specimens other than ovaries, ovarian non neoplastic lesions and also ovarian neoplastic specimens received before and after study period.

6.Methodology: As the department received good number of ovarian lesions regularly, the material was sufficient for this prospective study of the ovarian neoplastic lesions. With the help of case sheets and requisition forms particulars like age, clinical features, indication \& operative findings, clinical diagnosis were recorded. After receiving the specimen its weight, colour, shape, measurements and consistency like features were recorded. Then the lesion was cut opened and the cut surface was described. Adequate number of bits were taken from representative sites for processing and then paraffin embedding. Routine Haematoxyin and Eosin staining was done. Special stains like PAS and Reticulin etc., were used wherever needed[3,13]. A descriptive study of ovarian neoplastic lesions was done over a period of 3 years considering details of general incidence of ovarian lesions and general incidence of ovarian neoplastic lesions in particular, Age incidence of ovarian neoplastic lesions, unilaterality (right or left) or bilaterality were also made out. Macroscopic appearances and histopathological features and also common and rare lesions were described.

\section{Results}

During the study period of three years, total number of 7384 specimens were received for histopathological examination out of which 880 were ovarian lesions, constituting $11.92 \%$ of total received specimens in the department of Pathology, Siddhartha Medical College, Vijayawada.

In the present prospective study, out of 880 ovarian lesions, 92 were diagnosed as neoplastic lesions $(10.45 \%)$, which were included in the study. Out of 92, benign lesions were 66 and malignant lesions were 26 (7.50\% and $2.95 \%$ of all ovarian lesions respectively) as shown in Table I. No neoplasm was reported as borderline tumor. Benign lesions contribute a major part of neoplastic lesions with 66 of $92(71.74 \%)$ and remaining 26 of 92 (28.26) were malignant lesions. Various parameters like general and age incidences, uni or bilaterality, macro and microscopic features were discussed regarding overall neoplastic lesions and for benign and malignant lesions separately. Simple statistical tools were used in data analysis and presentation of results. Common features and histological types were discussed later.

\section{Discussion}

Present prospective study of ovarian neoplastic lesions with three years study period, 880 ovarian specimens were received (11.92\% of total received 7384 specimens) in Department of Pathology, Siddhartha Medical College, Vijayawada, Andhra Pradesh. In this part, important features of the present study are compared with the other similar studies[4,5].

\subsection{Age incidence}

In the present study, ovarian neoplastic lesions were observed in the range of 21 to 65 years age group with peak incidence between 31 to 50 yrs age group, covering $71 \%$ of the lesions. Ganga S. Pilli et al (2002) found the peak incidence of ovarian tumours in the $3 \mathrm{rd}$ and $4^{\text {th }}$ decades accounting 55.7\% [5]. Bhattacharya MM et al (1980) reported that the $2 / 3 \mathrm{rd}$ of the benign lesions were observed between 20 to $40 \mathrm{yrs}$ and $2 / 3^{\mathrm{rd}}$ of the malignant tumours were seen after 40 yrs age. Present study also showed the similar observations and correlates with the above studies. Ovarian malignancy accounted for 12.0 new female cases per 100,000 population in Australia 2000 (AIHW and AACR, AIHW National Mortality Database, Australia's Health 2004, AIHW) [6].

\subsection{Site incidence and Bilaterality}

In neoplastic lesions of ovary, out of total 92 lesions 64 lesions were unilateral and remaining 28 were bilateral lesions. Benign tumours were more unilateral than the malignant lesions. Out of 66 benign lesions, 48 lesions were unilateral (72.73\%) and remaining 18 lesions were bilateral (27.27\%). Malignant tumours were 26 out of which unilateral were 16 lesions $(61.54 \%)$ and bilateral were 10 lesions $(38.46 \%)$. Ganga S. Pilli et al 
reported $92.2 \%$ of the benign and $74.2 \%$ of the malignant tumours were unilateral[5]. Bhattacharya MM et al reported $85 \%$ of the neoplasms were unilateral and remaining $15 \%$ of the lesions were bilateral[4].

\subsection{Benign lesions}

Almost more than half of the benign lesions were covered under the group of surface epithelial tumors including surface epithelial serous, mucinous and also seromucinous tumors, shown in Table II. Out of 66 benign tumors, 48 were found as unilateral and the remaining 18 were bilateral tumors. Minimum age recorded was $21 y$ rs and the maximum was $58 y$ rs.

4.3.1. Surface epithelial serous tumors: In the present study, out of 66 benign lesions 26 were reported as surface epithelial serous tumors (39.39\% of the benign lesions) and it was the major part of overall neoplasms (28.26\%). Age of the patients varied from $21 \mathrm{yrs}$ to 60yrs, with a peak in 3rd and 4th decades. Few cases were partially solid with fibrous component or seromucinous type. Unilateralism is seen in 17 cases (65.38\%) and in 9 cases $(34.62 \%)$ bilaterality was seen. Majority of the tumors were more than $10 \mathrm{cms}$ in diameter in size, largest was of $32 \mathrm{cms}$ diameter found in a $60 \mathrm{yrs}$ old postmenopausal woman. It was smooth and with prominent vascular markings. On cut section the multiloculated cyst was filled with serous fluid and focal solid gray white areas were also observed. Microscopy gave the picture of serous cystadenofibroma.

4.3.2. Surface epithelial mucinous tumors: These have lower incidence rate than serous type. In this study, only 6 lesions were reported as mucinous type (9.09\%) of 66 benign lesions. Out of 6 lesions, 5 were unilateral and one was bilateral. These were observed in the age of 22 to 50 yrs. Already cut opened ovarian specimens received from a patients of 22 yrs and 36yrs of age and other specimen was from 35 yrs aged woman. Grossly all were multilocular and filled with mucoid material on their cut surfaces and no solid areas were found[7]. Microscopically the mucinous columnar epithelium confirmed them as mucinous tumors[8,14]. Few of the sections studied also showed the focal calcifications.

4.3.3. Benign Brenner tumors : The present study had 4 lesions (6.06\% of the benign lesions) reported as Benign Brenner tumors. Age of the patients was between 41 and 56yrs. All 4 lesions were unilateral with normal other ovary. One of these was $14 \mathrm{~cm} \times 11 \mathrm{~cm} \times 7 \mathrm{~cm}$ sized firm and nodular tumour. Cut section showed well circumscribed lobulated gray white area with focal yellow areas and small cystic areas. Microscopic picture of epithelial cell nests and surrounded fibrous ovarian stroma with focal glandular patterns suggestive of benign Brenner tumor[9].

4.3.4. Mature cystic teratoma: Out of 66 benign lesions, 14 were diagnosed as mature cystic teratomas ( $21.21 \%$ of the benign and $15 \%$ of all neoplasms). Of 14 lesions, 8 were unilateral and the remaining 6 were bilateral. Age distribution was from 24yrs to 50yrs with a peak in 3rd decade. These tumors were observed generally as globular masses, varying from $5 \mathrm{cms}$ in diameter to $9 \mathrm{x} 8 \mathrm{x} 6 \mathrm{cms}$ sized. Cut section, almost of all the tumors showed the pultacious material and also tufts of hair. Yellow greasy material, myxoid areas and dark brown areas were also found in some of the lesions in addition. Histology showed mainly the ectodermal elements like squamous epithelial lining and subepithelial adnexae like hair follicles. Myxoid areas in the gross were corresponded with cartilage microscopically and yellow areas were adipose tissue, both were components of mesoderm. Incidence of the present study is $15 \%$ and comparable with the above studies of Ganga S. Pilli et al and Bhattacharya MM et al [4,5].

4.3.5. Struma ovarii: Present study included 2 lesions reported as struma ovarii $(3.03 \%$ of the benign lesions). Both lesions were unilateral. Both lesions were observed as globular, firm and nodular gray white masses of $3 \mathrm{cms}$ and $4 \mathrm{cms}$ in diameter. Cut section showed solid gray white with focal brown areas and partial cystic changes. Microscopically thyroid acinar cells were demonstrated as follicles along with normal ovarian histology[9]. Thyroid follicles were filled with colloid and separated by thin fibrous septae.

4.3.6. Fibrothecoma: These are common in postmenopausal women. Only 5\% are bilateral. In the present study, 8 lesions were observed as fibrothecomas (12.12\% of benign). All were unilateral and the age ranged from $48 \mathrm{yrs}$ to $61 \mathrm{yrs}$. One lesion in $61 \mathrm{yrs}$ old patient was smaller $(3 \times 2 \times 1 \mathrm{cms}$ sized) and the other lesion in 48yrs aged patient was larger $(32 \times 22 \times 15 \mathrm{cms})$. Small lesions were solid gray white with small yellow areas. The larger lesions showed cystic areas with focal solid areas which were gray white and yellow. Consistency varied from soft to firm. Microscopy showed thecoma cells show pale vacuolated lipid rich cytoplasm and bland oval nuclei. Spindle cells are also seen representing fibrous component. In Leutinized thecoma, leutein cells were seen discrete and in nests.

4.3.7. Fibroma: As per WHO classification, fibromas comprise $4 \%$ all ovarian neoplasms with a mean age incidence of $48 \mathrm{yrs}$ and mostly bilateral[11,12]. In this study, 4 fibromas were reported $(9.09 \%$ of benign and $6.45 \%$ of overall neoplasms). Age incidence was from 48yrs to $53 y$ rs. One was bilateral (25\% of the fibromas). Size of the lesions varied from $1 \mathrm{~cm}$ diameter to $5 \times 3 \times 2 \mathrm{cms}$. Cut section showed solid and gray white picture in all lesions. Microscopy showed the fascicles of spindle cells with bland central oval nuclei. 
4.3.8. Surface fibroma: Only one lesion in present study was observed as surface fibroma in a patient of $26 \mathrm{yrs}$ age and it was unilateral (2.27\% of benign and $1.67 \%$ of overall neoplasms). Macroscopically it was a normal sized ovary with a cauliflower like firm growth on one end of the ovary externally. On cut section it was gray white, solid and firm nodule. Microscopically it was observed as fibroma as it contained fibroblasts arranged in fascicles.

\subsection{Malignant lesions}

Present study consists of 26 malignant lesions (28\% of neoplastic and 3.2\% of overall ovarian lesions). Unilateralism is observed in 10 lesions and remaining 6 lesions were bilateral. Age group ranging from 36yrs to $65 y r s$, denotes later age group than the benign lesions. Epithelial ovarian cancer was the fourth most frequent cause of death in women. Surface epithelial tumors are the most common of the ovarian tumors, accounting for $90 \%$ of all ovarian malignancies. In the present study, all 26 were the primary malignancies and the epithelial malignancies were 19 in number comprising $73.08 \%$ of the primary malignancies. The epithelial malignancies in the present study included 10 serous malignancies, 3 mucinous, 1 is seromucinous, 2 endometrioid type, 1 clear cell carcinoma and 2 transitional cell carcinomas as shown in table III.

4.4.1. Serous cystadenocarcinoma: In present study 10 were reported as serous cystadenocarcinomas (38.46\% of malignant and $10.87 \%$ of neoplasms). These were observed in the age group of $41 \mathrm{yrs}$ to $60 \mathrm{yrs}$. Six lesions were unilateral and 4 were bilateral. All the lesions were grossly large masses. The size varied from $4 \times 3 \times 3 \mathrm{cms}$ to $16 \times 16 \times 12 \mathrm{cms}$. All the lesions were uniloculated on cut section and three lesions showed papillary excrescences on inner surface. All the lesions were partly cystic and partly solid and had brown areas. Ragged inner surface was observed in one lesion. Histology showed the fallopian tube epithelium resemblence with stromal invasion and individual cell atypia[10]. Papillary tufting on cut section was seen in two cases. Extensive necrosis and congested blood vessels were present in almost all the lesions.

4.4.2. Mucinous cystadenocarcinoma: Three lesions in present study were reported as mucinous cystadenocarcinoma (11.5\% of malignant lesions). Two were unilateral and one was bilaterally present in a patient of 40yrs old. One side lesion was of $12 \mathrm{cms}$ diameter sized and the other side lesion was smaller with $5 \mathrm{cms}$ in diameter. Both were multilocular with cystic areas filled with mucinous material and the inner surfaces showed the papillary excrescences. Microscopically epithelium was endocervical type with malignant features. Omental secondary deposits of the tumor were observed in this case.

4.4.3. Seromucinous cystadenocarcinoma: In this present study, one lesion was reported as seromucinous cystadenocarcinoma, observed in 59yrs old patient (3.85\% of malignant lesions). The friable unilateral tumor of $6 \times 4 \times 3 \mathrm{cms}$ size was soft to cystic in consistency. Externally gray brown in color and the cut section showed myxoid material inside with focal solid gray white area. Histological combination of malignant serous and mucinous epithelium with papillary patterns was seen without vascular cores[11]. The other ovary was normal.

4.4.4.Endometrioid carcinoma: Two lesions(7.7\% of malignant lesions) were reported as endometrioid carcinoma in the present study. One was bilateral lesion with omental secondary deposits and the other lesion was unilateral. Grossly the tumors were cystic and solid masses of $7 \times 6 \times 4 \mathrm{cms}$ and $5 \times 5 \times 4 \mathrm{cms}$ sized lesions respectively. Cut sections showed cysts with thickened cyst wall, filled with dark brown fluid. Solid areas were firm, gray white and nodular. Microscopy showed the tumor resembling the proliferative endometrial cells with malignant features[15]. There were areas of hemorrhages and necrosis. Some areas showed sarcomatoid changes.

4.4.5. Clear cell carcinoma: Only one unilateral case of clear cell carcinoma was observed in 44yrs old patient (3.85\% of malignant lesions). It was solid, gray white tumor of $8 \mathrm{cms}$ diameter. Cut section showed cystic spaces with multiple yellow fleshy nodules. Histology comprised of polyhedral cells as sheets and papillary patterns. The individual cells showed abundant clear cytoplasm and malignant changes in the nuclei[16]. The epithelium was mimicking the gestational endometrium.

4.4.6.Transitional cell carcinoma: Present study had 2 reports of transitional cell carcinomas (7.7\% of malignant lesions). Both were unilateral lesions and grossly solid, yellow to tan colored masses of 10 and $12 \mathrm{cms}$ diameter. Microscopy showed the nested patterns of transitional epithelium in multilayered and papillary patterns[17]. Primary Transitional cell carcinoma of urinary bladder was excluded to rule out the possibility of secondaries in these cases.

4.4.7.Gynandroblastoma: It was as an unilateral lesion (3.85\% of malignant lesions) seen in 54yrs old woman. It was of $31 \times 28 \times 26 \mathrm{cms}$ sized multiloculated cyst with a solid area of $6 \times 4 \times 4 \mathrm{cms}$. Solid area was firm and gray white and dark brown in color. Cyst was filled with brown coloured fluid. Microscopy showed oval to spindle and polygonal cells arranged in hyper and hypocellular patterns. In the cellular areas, the spindle cells were arranged in sheets with intervening areas of polygonal cells with scanty cytoplasm and vesicular nuclei arranged in trabecular form, linear rows and well defined tubular forms, lined with tall columnar cells. Nuclear grooving was seen in some of the cells. Hypocellular areas showed spindle and polygonal cells with vacuolated 
clear cytoplasm. Mitotic activity was in a range of 4 to 6/10HPF. The histological picture was of mixed sexcord stromal malignant tumor showing all the four components and reported as Gynandroblastoma[18].

4.4.8. Granulosa cell tumor: Two out of 26 (7.7\%)malignant lesions were reported as Granulosa cell tumors, seen in $48 \mathrm{yrs}$ and $54 \mathrm{yrs}$ aged patients as unilateral lesions with other apparently normal ovary. Lesions were of $3 \times 3 \times 2 \mathrm{cms}$ and $4 \times 3 \times 3 \mathrm{cms}$ size respectively and grossly both were solid gray white with cystic areas[19]. Microscopically in both the lesions the tumor cells were arranged in sheets and trabecular patterns with characteristic focal Call-Exner bodies and nuclei showing longitudinal grooves ("coffee bean").

4.4.9. Yolk sac tumor: Second most common germ cell tumor type next to mature cystic teratoma occurs in childhood, adolescence and early adult life (mostly <30yrs). Also called as endodermal sinus tumor[20,21]. Present study included 2 unilateral lesions unilateral lesions (7.7\% of malignant lesions) of yolk sac tumors reported in $19 \mathrm{yrs}$ and $24 \mathrm{yrs}$ aged patients. Grossly they were of 9 to $11 \mathrm{cms}$ diameter sized masses. Cut sections were solid and cystic with hemorrhagic and necrotic areas. Microscopy gave the picture of cystic areas with flattened epithelial lining showing various degrees of atypia and also classical pattern of perivascular formations (Schiller-Duval bodies). Hence the lesions were reported as yolk sac tumors.

4.4.10.Poorly differentiated carcinoma: Two unilateral lesions (7.7\% of malignant lesions) were reported in 45yrs and 53 years old patients. Grossly gray-brown and solid. Microscopic picture of both showed pleomorphic epithelial cells with hyperchromatic nuclei infiltrating diffusely into adjacent adipose tissue and fibrous tissue alongwith areas of necrosis and hemorrhage. Tumor infiltration into ovarian stroma was also seen some areas. Thus picture is suggestive of poorly differentiated carcinoma infiltrating into ovarian stroma and fibrofatty tissue.

A recent study (W G McCluggage J.Clin. Pathol 2008) from Washington of consecutive cases of ovarian carcinoma operated in a large hospital (included ovarian carcinosarcomas and primary peritoneal carcinomas, particularly all with an epithelial component of serous type) showed a different frequency of the various types of ovarian carcinoma. In that study, $68 \%$ were ovarian carcinomas of serous in type. The next common was clear cell $(13 \%)$, followed by endometrioid and mucinous (9\% and $3 \%$ respectively). Six per cent of tumours were of mixed type and only $1 \%$ were transitional carcinomas.

In a study conducted by MM Bhatacharya et. $\mathrm{Al}[4]$, there were 17 sex cord stromal tumours forming about $6.80 \%$ of all ovarian neoplasms. Granulosa cell tumours numbered 8 out of which 2 were benign and 6 were malignant. Thecomas constituted 2; fibromas, 4; fibrothecomas were two and androblastoma was only one.

In a study(by Kataoka et al , Nippon Sanka Fujinka Gakkai Zashi.1989)[20] of malignant germ cell tumors at Kurume University Hospital, for 18 years, the total number of patients was 112, including 25 yolk sac tumors (YST), 10 mixed form germ cell tumors (MF), 36 dysgerminomas (DYS), 26 immature teratomas (IT), 2 choriocarcinomas $(\mathrm{CHO})$ and 13 dermoid cysts with malignant transformation (DCMT). When DCMT cases were excluded, ages was closely related to the age of menarche in each patient.

The incidence, clinical appearance and the behavior of the different types of ovarian tumours is extremely variable. It is generally difficult to diagnose the nature of the ovarian tumour preoperatively just by clinical examination and even on exploration, though certain investigations like peritoneal fluid cytology, estimation of serum lactic dehydrogenase, fibrin degradation products and immunological tests have been reported to be of some help in predicting the nature of the pathology. Hence one has to depend on the microscopic appearance of the tumor for management of the ovarian neoplasms[4].

\subsection{Radiotherapy(RT)}

Radiotherapy was used as main line of management in certain type of ovarian cancers like Dysgerminomas which showed tremendous response[22]. But because of infertility caused by radiotherapy gradually being replaced by chemotherapy, except in such cases still radiotherapy is the line of management[23]. There was no improvement in survival in people with well differentiated ovarian cancers[23].

Radiotherapy was also used as part of palliative care of patients with cancers of advanced stage as helped in reducing the size of tumor which caused symptoms due to its larger size. This palliative RT was of shorter course when compared to non palliative RT course[24]. This was also used in chemotherapy resistant germ cell tumors[25]. 


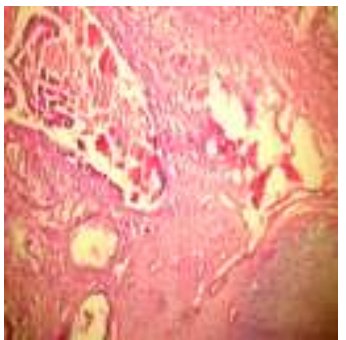

Fig.1

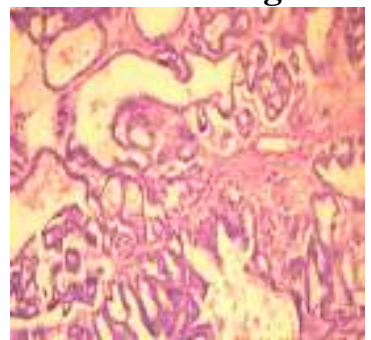

Fig.2

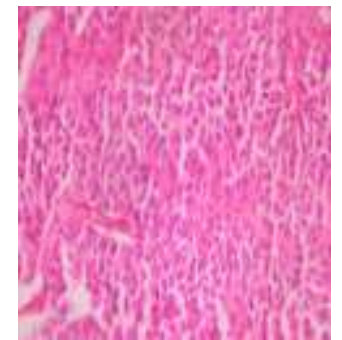

Fig.3

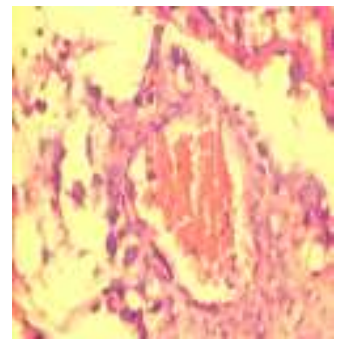

Fig.4

Mature cystic teratoma

Papillary serous cystadeno carcinoma

Granulosa Cell Tumor Yolk sac tumor

Table I: Distribution of Ovarian lesions

Non Neoplastic, 788

Neoplastic- Benign,66

Neoplastic- alignant, 26

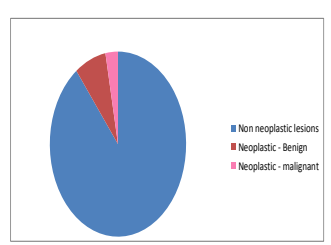

Table II: Distribution of benign ovarian lesions

- Surface epithelial

tumour Serous - 26

- Surface epithelial

tumour Mucinous -06

- Mature cystic

Teratomas - 14

- Fibrothecoma -

- Fibroma -

- Surface Fi

- Brenner Tumour - 04

- Struma Ovarii - 02

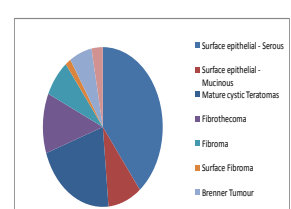

Table III Distribution of malignant ovarian lesions

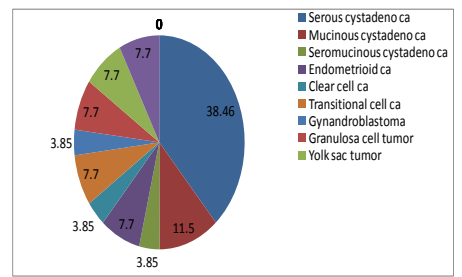

\section{Conclusions}

Ovarian neoplasms are one of the common types found in the women of reproductive age group. Accurate diagnosis and also typing \& grading according to recent and standard classifications like WHO Classification is useful in the line of management as specific therapies, like chemo/ surgical/ radiotherapies including targeted therapies or combination of the various therapies depending on the type, stage and grade of ovarian cancer. An attempt was made to study the age incidence, prevalence, morphological types and histological variants among various ovarian neoplastic lesions. The results of the study are comparable with other similar studies and standard books substantiating the findings of the study. About 646 ovaries showed normal histology and no specific pathological changes noted. This may be due to the precautionary removal of ovaries at the time of hysterectomy to avoid further risk or similar surgery. There is a possibility of hidden minute pathology in these which had not appeared in the sections studied as the total ovary was not being processed and studied. Role of radiotherapy in ovarian neoplasms is also discussed.

\section{References}

[1]. Fattaneh A, Tavassoli, Peter Devilee; WHO Classification of Tumours: Pathology and genetics of tumours of the breast and Female genital organs-2003; page-113- 202.

[2]. Tandon, R., Mathur, J.S., Mehram, M., Sharma, U: Pattern of ovarian tumors in Ajmeer (Rajasthan) J. Ind. Med. Ass. 77 : 191-193, 1981.

[3]. Bancroft's Theory and Practice of Histological Techniques, 7th edition(2013) pages 80 to 163.

[4]. Bhattacharya MM, Shinde SD, Purandare VN; A Clinicopathological analysis of 270 ovarian tumours, Journal of postgraduate Medicine, Volume-26, issue-2, page 103- 7, 1980

[5]. Ganga S Pilli,K P Suneeta, A V Dhaded, V V Yenni; Ovarian tumours; A Study of 282 cases, JIMA,VOL-100,July 2002, page no420-424.

[6]. Morris, J.M., Scully, R.E. : Endocrine Pathology of the ovary. St. Lowis, 1958.

[7]. Prat, J., Young, R.H., Scully, R.E. : Ovarian mucinous tumors with foci of Anaplastic carcinoma. Cancer 50: 300 - 304,1982

[8]. diFiore's Atlas of Histology, by victor P. Eroschenko: 11th edition, 2008

[9]. Robbins and Cotran Pathologic Basis of disease:Kumar,Abbas,Fausto, 9 th ed 2014, page: 1022 to 1034

[10]. Anderson's Pathology, Ivan Damjanov, James Linder, 10th edition 1996.Elsevier.

[11]. Blaustein's pathology of the female genital tract, 6th edition, Kurman. Robert J. (20110, page no-677-928.

[12]. Fritz A, Percy C, Jack A,Shanmugaratnam K, Sobin LH,Parkin DM,Whelan S (2000).International classification of diseases for oncology(ICD-0) $3^{\mathrm{RD}} \mathrm{ED}, \mathrm{WHO}-G e n e v a$.

[13]. Lynch's Medical Laboratory technology, Stanley S. Raphael, Third edition.

[14]. Langman's Medical embryology by T.W.Sadler: $9^{\text {th }}$ edition,2004,Development of urogenital system, Page no-270-281.

[15]. S Maharjan, Clinicomorphological Study Of Ovarian Lesions, Journal of Chitwan Medical College 2013; 3(6): 17- 24

[16]. Shaw's text book of Gynaecology. Ed.Padubidri V.G. Shirish N. Daftary. $14^{\text {th }}$ Edition, B.I. Churchill Livingstone, New Delhi, 2008: pages 329 to 349

[17]. Steven G. Silverberg - principles and practice of surgical pathology and cytopathology 4th edition. Churchill Livingston 2006: page no- 1987 to 2039 
[18]. Rosai J. Rosai and Ackerman's surgical pathology. 9thed. St. Louis: CV Mosby Co.; 2004. vol 2:1649-1736

[19]. Kanthikar S.N. et al., Clinico-Pathological Study of Neoplastic and Non-Neoplastic Ovarian Lesion, www.jcdr.net, , 2014 Aug, Vol-8(8): FC04-FC07

[20]. Kataoka A,Yakushiji M, Nishida T,Oda T, Kojiro M; Clinicopathological study of malignant ovarian germ cell tumours; 1989.

[21]. Sahu L., Mallik R.N., Nanda M., Sethy C.R. and Dash S Germ cell tumours of ovary - Review of 46 cases J.Obstet \& Gynaec. India 40: 446 - 450, 1990

[22]. DeCherney, Alan; Nathan, Lauren; Goodwin, T. Murphy; Laufer, Neri; Roman, Ashley (2012). "Pediatric and Adolescent Gynecology". Current Diagnosis \& Treatment Obstetrics \& Gynecology (11th ed.). ISBN 978-0071638562

[23]. Seiden MV (2012). "Gynecologic Malignancies". In Longo DL, Kasper DL, Jameson JL, Fauci AS, Hauser SL, Loscalzo J. Harrison's Principles of Internal Medicine (18th ed.). McGraw-Hill. ISBN 978-0-07-174889-6.

[24]. "Radiotherapy for ovarian cancer". www.cancerresearchuk.org. Retrieved 2015-05-16.

[25]. Hoffman BL, Schorge JO, Schaffer JI, Halvorson LM, Bradshaw KD, Cunningham F, Calver LE (2012). Ovarian Germ Cell and Sex Cord-Stromal Tumors. Williams Gynecology (2nded.) (McGraw Hill). 